\title{
Design of High Precision Electronic Scale Based on MSP430G2
}

\author{
Jian Huang \\ Xijing University, Xi'an 710123, China \\ 565200245@qq.com
}

Keywords: Small signal; Instrumentation amplifier; High precision A/D; MSP430G2553

\begin{abstract}
The resistance bridge is used as a pressure sensor, when the external force is applied to generate $0 \sim 20 \mathrm{mV}$ voltage small signal, the precision instrumentation amplifier amplification 51 times, access to 16 bit high precision digital analog conversion (A/D) chip ADS1146. 16 bit A/D in the input voltage of $3.6 \mathrm{~V}$, the minimum resolution of $0.05 \mathrm{mV}$, to achieve high precision pressure sensor signal acquisition, the accuracy can reach 0.1 grams. The A / D conversion to digital quantity through the SPI interface connected to the low power microprocessor msp430g2553, and then sent to the LCD display, through the calibration button and do some simple multiplication and achieve more functional. Test results show that the small signal amplification link can achieve the stability of the signal amplification, and can achieve accurate weighing.
\end{abstract}

\section{Introduction}

How to detect the weak electrical signal is a problem that the hardware circuit designer must consider. In real life, we often to detect the pressure signal. Usually the pressure sensor is a resistance bridge, there are four internal resistance. When there is an external force and deformation sensor. Under the external force of bending, and the upper end of the elongated, lower pressure was short, one end of the elongated with proportional corresponding resistance value, stub end in negative proportion to reduce the corresponding resistance value. Because of the changes in resistance, the voltage output of a bridge pressure is $0 \sim 20 \mathrm{mv}$. To collect the voltage must be to carry on the effective amplification, early amplified when people used LM324 op amp amplification cannot collect signals effectively. And interference particularly large, accuracy is not up to the requirements.

In this design, a new type of high precision instrumentation amplifier INA128 is used to amplify the signal by 51 times, which has high common mode rejection ratio, and can effectively restrain the interference by using differential input. The amplified signal is connected to the high precision 16 bit A/D for signal acquisition, and the converted signal is connected to the low power consumption microprocessor MSP430G2553 for processing. Can achieve high precision weighing.

\section{System Design}

The system block diagram is shown in Fig. 1. The small signal sensor circuit can be a pressure sensor, ultrasonic sensor and other sensors. Instrument amplifier using TI high precision instrumentation amplifier INA128, when the input signal $200 \mathrm{KHz}$, the gain of G can reach 100 , its common mode rejection ratio of CMRR reached $120 \mathrm{~dB}$, can effectively suppress the interference signal. Negative pressure generation circuit provides $-3.6 \mathrm{~V}$ power supply voltage for INA128. In the circuit design, INA128 can amplify the input signal by 51 times. Then input to high precision 16 bit A/D conversion chip ADS1146.

ADS1146 is a high precision, low noise, 16 bit A/D converter, which contains a programmable gain control module (PGA), amplification ratio of 1, 2, 4, 8, 16, 32, 64, 128 adjustable. Built in reference voltage, and can be output two DA. The analog voltage of the instrument amplifier can be converted to digital quantity, and the microprocessor can be transmitted to the microprocessor through the SPI interface. The microprocessor uses the TI low power processor MSP430G 2553, the key circuit can carry on the school zero to the acquisition voltage, or does some simple operation, displays the collection weight and other information on the display circuit. 


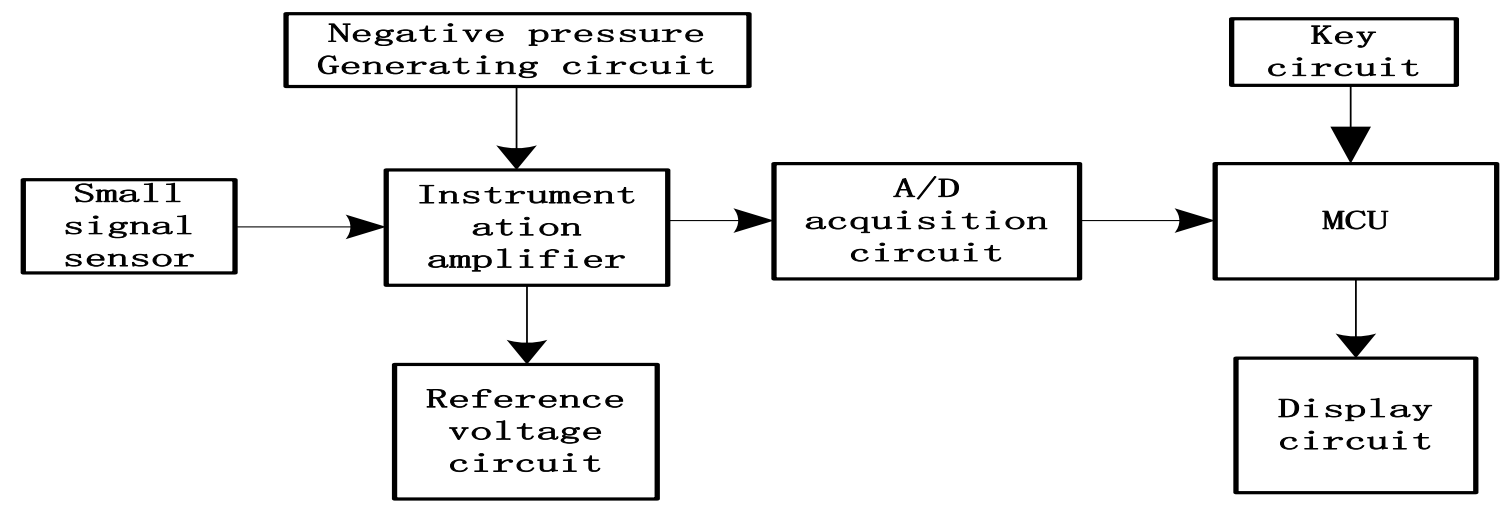

Figure 1. System design

\section{Detailed Design and Key Technologies}

Pressure Bridge and Instrumentation Amplifier Circuit Design. Pressure bridge and instrumentation amplifier circuit is shown in Fig. 2. The equivalent circuit of the bridge pressure such as Fig. 2 (left) shows, consisting of two fixed resistor R and a variable resistance R1 and R2, when in the absence of force, bridge balance and pin 1 of the output voltage is VCC/2. 2 feet of the output voltage is $\mathrm{R} 2 * \mathrm{VCC} /(\mathrm{R} 1+\mathrm{R} 2)$. Pressure sensor is real graph abstraction as shown in the diagram in Fig. 2 A, when in the absence of force, face F1 and F2 isometric; when a heavy into F1, support due to the pressure sensor on the left, the whole will bend and on the surface of F1 is elongated, will cause the R1 resistance to certain ratio increased, surface F2 is pressed short, will cause resistance $\mathrm{R} 2$ to reduce certain proportion. This will cause the output voltage value of the 2 pin to change. Pressure sensor is usually full range of 1 feet and 2 pin differential output voltage range is $0 \sim 20 \mathrm{mv}$. This signal must be amplified, in the design of instrumentation amplifier INA128 amplifier.

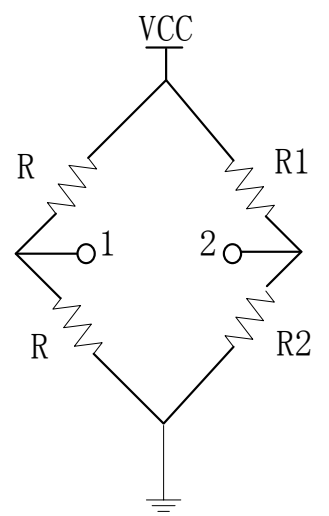

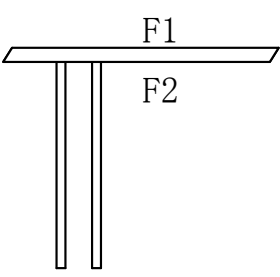

图A

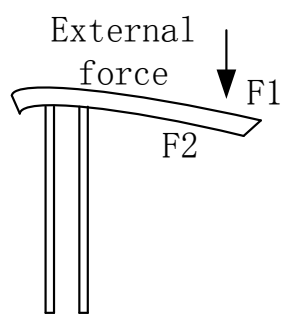

图B

Figure 2. Pressure bridge and its equivalent circuit

INA128 simulation circuit is shown in Fig. 3. The differential input, bridge pressure generated voltage access ina128 2 feet (in -) and 3 feet (+). Between pins 1 and 8 access resistance $\operatorname{Rg} .5$ foot access reference voltage $\mathrm{V}_{\text {ref, }}$, when power supply film is $3.6 \mathrm{~V}, 3$ shown TL431 can output stable $1.8 \mathrm{~V}$ voltage. Negative pressure generated by TPS60400. The calculation of the magnification of the INA128, as shown in the formula (1), the calculation of the output voltage, as shown in the formula (2).

$$
\begin{aligned}
& G=1+\frac{50 \mathrm{~K}}{R_{g}} \\
& V_{O}=\left(V_{1}-V_{2}\right) \times G+V_{r e f}
\end{aligned}
$$




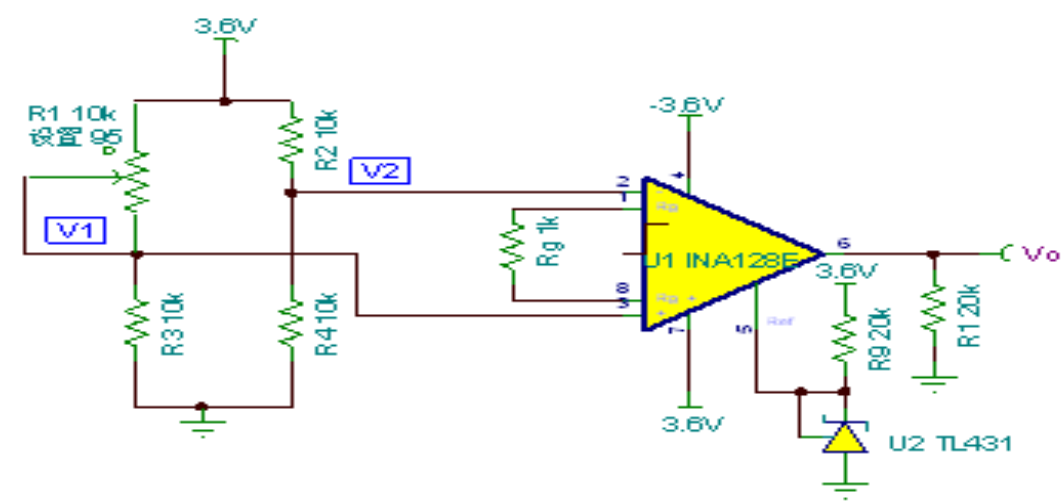

Figure 3. Pressure bridge and instrumentation amplifier circuit

A/D Acquisition Circuit Design. In this design, the filter circuit is omitted between the instrument amplifier and the A/D acquisition circuit. At present, the latest signal chain design circuit, the A/D, filter, PGA, D/A is often integrated in a chip. And ADS1146 is a new type of high precision $\mathrm{A} / \mathrm{D}$ acquisition chip. Its pin figure as shown in Fig. 6, the use of 3.6V power supply, 16 bit $\mathrm{A} / \mathrm{D}$ can reach the minimum resolution of $3.6 \mathrm{~V} / 65536=0.05 \mathrm{mV}$, enough to meet the demand. The INA128 amplified voltage input ADS1146 7 feet (AINP) as the analog input, the digital output after the conversion through the SPI bus, that is, ADS1146 14 feet (DOUT) and 15 feet (DIN) connected with the MSP430G2553.

MSP430G2553 and Its Peripheral Circuit Design. In the design of the circuit in the third pin (P11) and fourth pin (P12) as the SPI bus, P20, P21, P22 to do 12864 LCD display circuit serial data input. P17 can be used as the A/D input interface, the detection of key input values.

12864 display circuit design

Display driver circuit as shown in Fig. 7, 12864 is graphics and text LCD, due to msp430g2553 pin Co., so the two 74HC595 serial to parallel conversion chip, driving 12864 .HC595 can msp430g2553 P22 pin input serial data through the parallel interface QA to QH output to 12864.

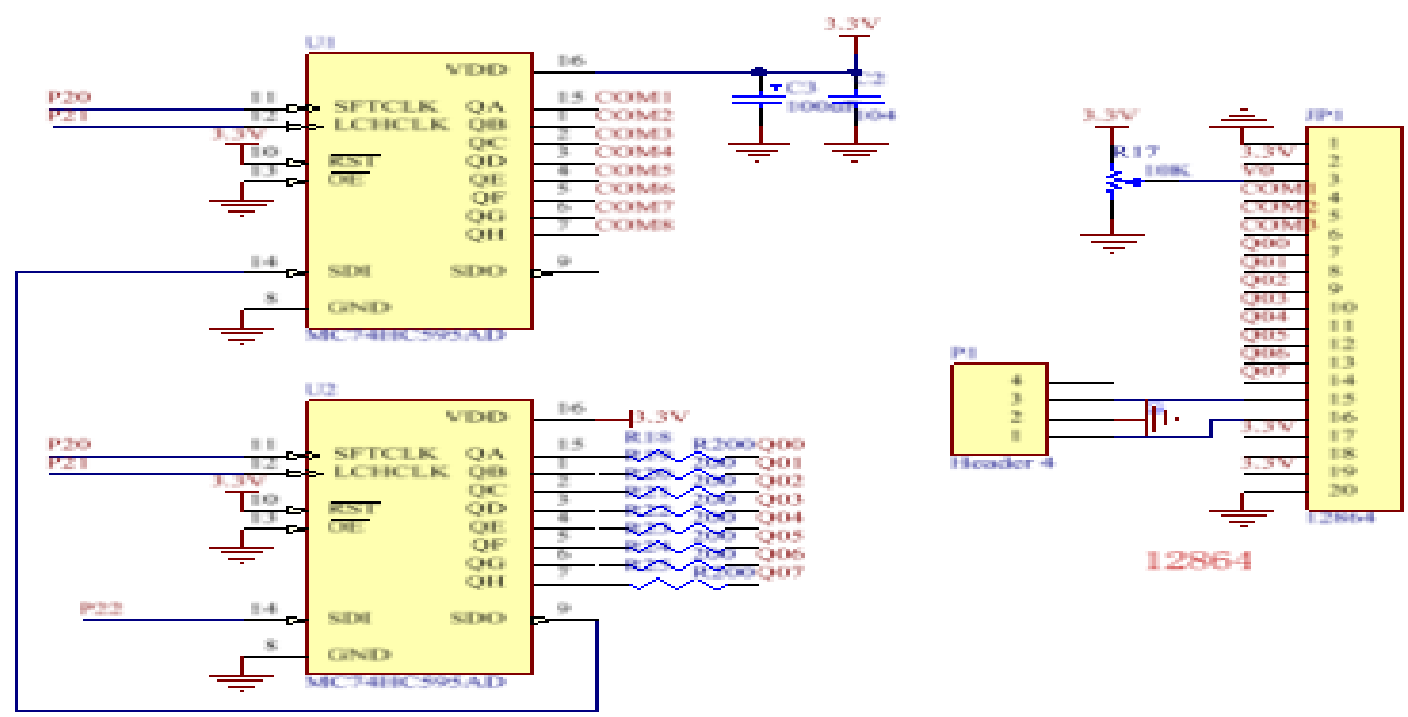

Figure 4. 12864 shows the driving circuit

\section{Simulation and Test Results}

At first, the output signal of the pressure sensor is tested in the simulation. Test, first of all to $+3.6 \mathrm{~V}$ voltage four wire pressure sensor red and black two lines, then the weight from 1 to 200 grams in turn add to the test platform, test pressure sensor of green, white two output line voltage. We can get the data shown in Table 1. 
Table 1 The relationship between the output voltage and the weight of the pressure sensor

\begin{tabular}{|l|l|l|l|l|l|l|l|l|l|l|}
\hline height $(\mathrm{g})$ & 1 & 2 & 3 & 10 & 53 & 193 & 458 & 1050 & 2000 & 5529 \\
\hline voltage $(\mathrm{mv})$ & 0.001 & 0.002 & 0.003 & 0.01 & 0.046 & 0.167 & 0.398 & 0.869 & 1.693 & 4.682 \\
\hline
\end{tabular}

The correspondence between the measured weight and the actual conversion is shown in Table 2 after the design of the software. As can be seen from the table, the error in the $0.1 \mathrm{~g}$ or so, basically meet the design requirements.

Table 2 The corresponding table between the measured weight and the actual value

\begin{tabular}{|l|l|l|l|l|l|l|l|l|l|l|}
\hline Actual weight (g) & 1 & 2 & 3 & 10 & 50 & 100 & 200 & 1000 & 2000 & 5000 \\
\hline Measuring weight (g) & 1.1 & 2.1 & 2.9 & 9.9 & 49.8 & 100.1 & 200.2 & 1000.1 & 2000.2 & 4999.8 \\
\hline
\end{tabular}

\section{Conclusions}

In this paper, a high precision instrumentation amplifier for small signal amplification of the pressure sensor data link, due to the use of differential input, effective suppression of interference, eliminating the filter circuit design. After simulation and test, the circuit can realize the effective amplification of small signal. 16 bit A/D can be used to improve the accuracy of the acquisition. The test results show that the high precision acquisition of small signal is realized, and the accuracy is 0.1 grams. Has a certain practical reference value.

\section{References}

[1] Texas Instruments. ADS1146 [M]. Shanghai, 2012, 4.

[2] Texas Instruments. INA128 [M]. Shanghai, 2005, 6.

[3] Texas Instruments. TINA - TI quick start guide [M]. Shanghai, 2008, 5.

[4] He ping; Guo-feng pan; Sun to material; Hong-dong zhao; Zhang Yinhui. RBF network model of pressure sensor temperature drift compensation $[\mathrm{J}]$. Journal of instruments and meters, 2008, $29(3): 572-576$

[5] Lv Haojie; Guo-qing hu; Zou Wei; Can-yun wu; Chen Yu front. The design of the high-performance MEMS capacitive pressure sensor and its thermal analysis [J]. Optical precision engineering, 2010, 17 (5) : 1166-1173

[6] Xiao - li zhang, shui-jin Chen. The status and development of resistance to high temperature pressure sensor [J]. Journal of sensors and micro systems, 2011, 30 (2) : 1-4

[7] Hou Peiguo, XingYao double hai-lan yao, li pan. Low-power wireless pressure transmitter based on MSP430 research and design [J]. Instrument technique and sensor, 2011, 2 (2) : 19 to 25

[8] zhang, re-evaluation, Ding Mingdong PangXing. Based on the MSP430 MCU and high precision pressure transmitter [J]. Instrument technique and sensor, 2010, 1:32-35

[9] TongShiBai, hua cheng ying. Analog electronic technology foundation [M]. Beijing: higher education press. 2009

[10] Texas Instruments. Texas Instruments of high performance simulator for a college application guide [M]. Shanghai, 2014, 3. 